\title{
Prognostic significance of intraoperative change in the fractional anisotropy and the volume of the optic chiasma during resection of suprasellar tumors
}

\author{
Hussam Metwali, MD, MSc, Mario Giordano, MD, Katja Kniese, MD, and \\ Rudolf Fahlbusch, MD, PhD \\ International Neuroscience Institute, Hannover, Germany
}

OBJECTIVE The aim of this study was to test the prognostic significance of intraoperative changes in the fractional anisotropy (FA) and the volume of the optic chiasma and their correlation with visual outcome.

METHODS Twenty-eight sequential patients with suprasellar tumors presenting with chiasma compression syndrome were surgically treated under intraoperative MRI control between March 2014 and July 2016. The FA and the volume of the optic chiasma were measured immediately before and immediately after tumor resection. The visual impairment score (VIS) was used to quantify the severity of the ophthalmological disturbances before surgery, 10-14 days after surgery, and again 3 months thereafter. The change in the FA and the volume of the optic chiasma was correlated to the improvement of vision. The correlation between other predictors such as the age of the patients and the duration of symptoms and the visual outcome was tested.

RESULTS The VIS improved significantly after surgery. The FA values of the optic chiasma decreased significantly after decompression, whereas the volume of the optic chiasma increased significantly after decompression. The early and delayed improvement of vision was strongly correlated to the decrease in the average FA and the increase of the volume of the optic chiasma. The duration of symptoms showed a significant negative correlation to the visual outcome. However, the decrease in the FA showed the strongest correlation to the improvement of the VIS, followed by the expansion of the optic chiasma, and then the duration of symptoms.

CONCLUSIONS The decrease in the FA and the expansion of the optic chiasma after its decompression are strong early predictors of the visual outcome. These parameters are also able to predict delayed improvement of vision.

https://thejns.org/doi/abs/10.3171/2016.12.JNS162741

KEY WORDS fractional anisotropy; intraoperative MRI; visual outcome; suprasellar; diagnostic technique; pituitary surgery

$\mathrm{S}$ UPRASELlar tumors tend to compress the optic chiasma and to cause visual impairment in the form of visual field defects and decreased visual acuity. Decompression of the optic chiasma can improve the visual impairment. In cases of long-term chronic compression, the recovery of the visual acuity and field of vision is limited or even absent due to permanent chronic changes of the optic chiasma. ${ }^{1,15}$

The MR anatomical imaging offers an idea of the degree of the compression and displacement of the optic chiasma. Fiber tracking based on diffusion tensor imaging (DTI) can improve the visualization of the optic pathways. Besides the possibility of reconstruction of the visual pathways via fiber tracking, DTI can provide further useful measurements like fractional anisotropy (FA), which can be considered as an index of the integrity of the optic pathway. It has been found that the FA of the optic pathways could be affected in diverse ophthalmological conditions. ${ }^{8,9,25}$ Furthermore, changes in the FA have also been found to be related to different diseases of the CNS, their progression, and their response to treatment. . $^{4,8,9,11,17,22,32,37}$

In this study, we focused on the intraoperative change in the FA and the volume of the optic chiasma after its decompression in patients harboring suprasellar tumors that were treated under intraoperative MRI control. We correlated the change of the FA and the volume of the optic chiasma to the improvement of the visual status. To the best of our knowledge, this is the first study to discuss the significance of the intraoperative changes in the FA and the volume of the optic chiasma as very early predictors of

ABBREVIATIONS DTI = diffusion tensor imaging; FA = fractional anisotropy; VIS = visual impairment score .

SUBMITTED October 30, 2016. ACCEPTED December 21, 2016.

INCLUDE WHEN CITING Published online June 23, 2017; DOI: 10.3171/2016.12.JNS162741. 
the visual outcome. We tested the correlation of these parameters to the delayed visual outcome as well. We evaluated other factors than can affect the visual outcome, like the age of the patients and the duration of symptoms, to determine which factor had the strongest correlation to the visual outcome.

\section{Methods}

We performed a retrospective analysis of the patients in whom suprasellar tumors were resected and who presented clinically with visual impairment and radiologically with chiasma compression. The patients were surgically treated between March 2013 and July 2016 under intraoperative MRI control. The DTI of the visual pathway was performed before and after tumor resection. The volume of the optic chiasma was measured before and after tumor resection. All patients underwent ophthalmological evaluation before surgery, 10-14 days after surgery, and 3 months thereafter.

\section{Assessment of the FA}

All the patients were treated under 1.5-T intraoperative MRI control (Magnetom Espree; Siemens AG Medical Solution [software NUMARIS/4, version syngo MR B17]). The head was fixed using an MRI-compatible head fixator. An initial intraoperative MRI study (before tumor resection) was performed in all patients, including volumetric anatomical imaging (T1 weighted) as well as DTI (resolution $128 \times 128 \times 60$ voxels, voxel size $1.875 \times 1.875$ $\times 2.7 \mathrm{~mm}, 20$ directions, and $b$ values of $1000 \mathrm{sec} / \mathrm{mm}^{2}$ ). The images were then transferred to the planning workstation (iPlan; Brainlab AG). The images (T1-weighted and DT) were fused. The optic chiasma was segmented manually on the volumetric T1-weighted images-which were fused with the DT images-under magnification by using a segmentation brush. The segmentation was strictly inside the chiasma to avoid contamination of the region of interest with CSF or tumor tissue, which would affect the FA values (Fig. 1A-D). The FA value of the segmented optic chiasma was measured (minimum, maximum, and average). The volume of the optic chiasma was calculated. After tumor resection, a control MRI session including T1-weighted and DTI was performed with the same head position, sequences, gantry tilt, and setting as the initial MRI. The images-T1-weighted and DT-were fused and the optic chiasma in the new image set was segmented (Fig. 1E-H), and then the FA values and the volume of the optic chiasma were measured.

In all cases we used the right cerebral peduncle as a reference point. The cerebral peduncle was segmented on the intraoperative volumetric T1-weighted images, which were fused with the intraoperative DT images acquired before tumor resection, and on the control studies obtained after tumor resection. The FA values were then measured to exclude the possibility of technique-related changes in the FA.

\section{Assessment of Visual Outcome}

Ophthalmological evaluation was performed in all patients before surgery, 10-14 days after surgery, and 3
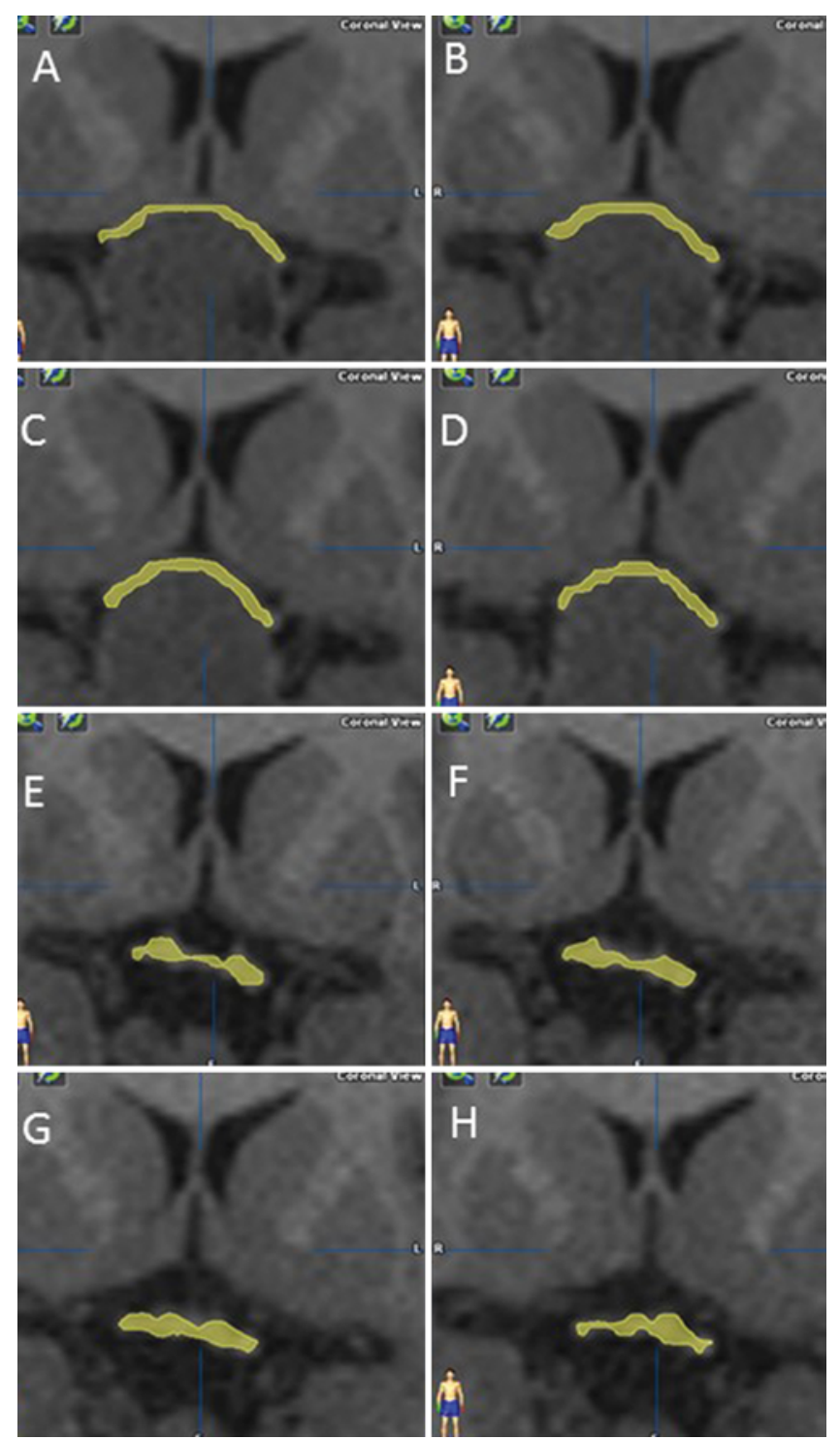

FIG. 1. A-D: Fused T1-weighted and DT images showing the segmented optic chiasma before tumor resection. E-H: Fused T1-weighted and DT images showing the segmented optic chiasma after tumor resection. Segmentation was performed on volumetric T1-weighted images. Figure is available in color online only.

months thereafter. The visual acuity, funduscopy, intraocular pressure, and field of vision were assessed by an independent ophthalmologist. The visual acuity was assessed using Snellen's chart and the field of vision was assessed using a Goldmann and Humphrey field analyzer.

To convert the visual status into a numerical value, we calculated the visual impairment score (VIS) preoperatively and postoperatively according to the guidelines of the German Ophthalmological Society. ${ }^{12}$ The field of vision was calculated for both eyes, as was the visual acuity. Each degree of defects was assigned a special score. The scores for visual acuity and visual field defects in each patient were added together to calculate the VIS. Examples of the chart and the calculation are described in the illustrative case. 
The assessors of the FA and the volume of the optic chiasma were blinded to visual outcome. The assessors of the visual outcome were blinded to the FA and the volume of the optic chiasma. The analysis was done with pseudoanonymous data.

\section{Statistical Analysis}

The statistical analysis was performed using SPSS version 20 (IBM Corp.). The normality of the data distribution was tested with the Shapiro-Wilk test. Because the data were not normally distributed and the sample size was relatively small, we used Spearman's rank correlation coefficient to test the correlation. The correlation coefficient was calculated to the visual outcome for each predictor in this study (the change in the FA, the change in the volume, the duration of symptoms, and the age of the patients).

The Wilcoxon signed-rank test was used to compare the VIS and the FA before and after surgery. The difference between the preoperative and the postoperative measurements and score were considered significant at $\mathrm{p} \leq$ 0.05 . Because the data were not normally distributed, we used the $\mathrm{W}$-value to assess the significance.

\section{Results}

Twenty-eight sequential patients were included in this study. The group included 8 women and 20 men. The age ranged from 30 to 72 years (average 53 years). Twentythree patients suffered from pituitary adenomas, 3 patients from craniopharyngiomas, 1 patient from Rathke cleft cyst, and 1 patient from suprasellar meningioma. The duration of symptoms ranged from 2 weeks to 4 months. Twenty-six patients were treated via the transsphenoidal approach, and 2 were treated via the right frontolateral approach. There were no documented vascular or mechanical injuries in any of the patients.

\section{Change in the Average FA Values}

The average FA values of the optic chiasma decreased after decompression of the optic chiasma in 24 patients and remained unchanged in 4 patients. The change in the average FA after operative decompression ranged from 0 to 0.32 (mean 0.1, SD 0.8). The FA of the reference region of interest (the right cerebral peduncle) did not show change between the images obtained before and after tumor resection.

\section{Visual Outcome}

The preoperative VIS ranged from 2 to 44 (mean 14.8, SD 10.8). The postoperative score ranged from 0 to 30 (mean 8.7, SD 7.8). The VIS after surgery was improved in 24 patients, unchanged in 2 patients, and worse in 2 patients. The improvement was recorded as a positive value and the deterioration as a negative value compared with the preoperative score. The change in the VIS ranged from -8 to 30 (mean 6.2, SD 8.7).

The visual evaluation was available in 26 patients after 3 months and showed further improvement. The VIS ranged from 0 to 20 (mean 3.2) after 3 months. One patient with deteriorated vision after surgery showed improve-

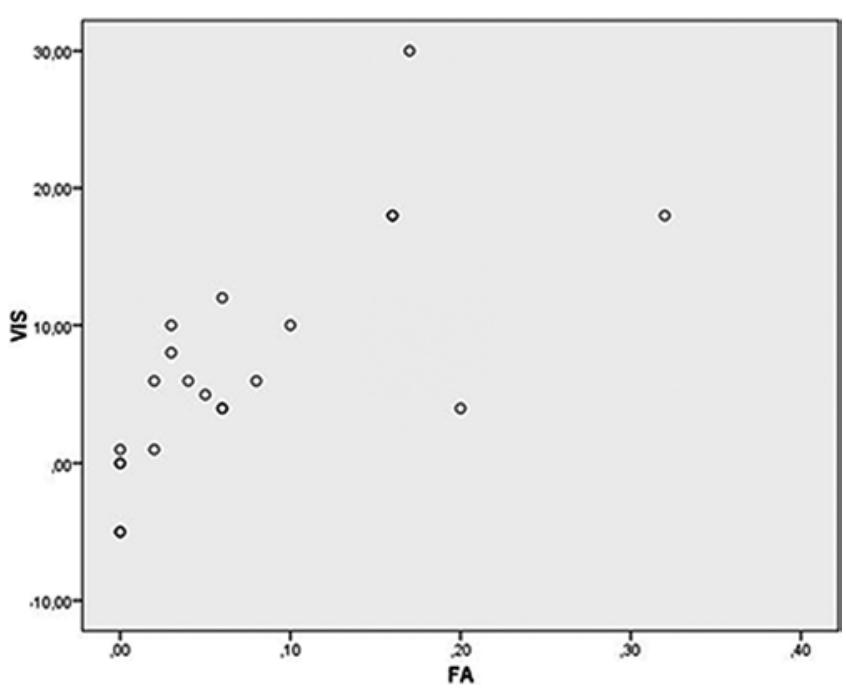

FIG. 2. A scatter plot showing the correlation between the decrease in the FA ( $x$ axis) and the improvement of the VIS (y axis).

ment of his VIS to a level better than his preoperative VIS after 3 months. This patient showed an intraoperative decrease in the FA. Unfortunately, the other patient with deteriorated VIS after surgery and unchanged intraoperative FA did not show improvement after 3 months. The 2 patients with unchanged visual status after surgery and intraoperative decrease in the FA showed improvement in the VIS after 3 months.

\section{Immediate Expansion of the Optic Chiasma After Decompression}

The volume of the optic chiasma expanded in 22 patients and remained unchanged in 6 patients. The increase in the volume ranged from 0 to $0.03 \mathrm{~cm}^{3}$.

\section{Correlation Between Change in the FA and Volume of the Optic Chiasma and the VIS}

FA of the Optic Chiasma

The FA values of the optic chiasma decreased significantly after decompression, as shown by the Wilcoxon signed-rank test at $\mathrm{p} \leq 0.05$. (The $\mathrm{W}$-value was 0 . The critical value of $\mathrm{W}$ for $\mathrm{N}=16$ at $\mathrm{p} \leq 0.05$ was 29.) The VIS improved significantly after surgery, as assessed by the Wilcoxon signed-rank test at $\mathrm{p} \leq 0.05$. (The W-value was 16 . The critical value of $\mathrm{W}$ for $\mathrm{N}=19$ at $\mathrm{p} \leq 0.05$ was 46).

The improvement of the VIS was strongly correlated to the decrease in the average FA values of the optic chiasma after its decompression (Spearman's rank correlation coefficient 0.76, significance level [2-tailed] < 0.0001) (Fig. 2 ). The decrease in the FA was also significantly correlated to the further improvement of the VIS after 3 months.

There was no significant correlation between the preoperative average FA values and the preoperative VIS (correlation coefficient 0.13, significance level [2-tailed] $0.43)$. The postoperative VIS was not correlated with the postoperative FA values (correlation coefficient 0.09 , significance level [2-tailed] 0.6). 


\begin{tabular}{|c|c|c|c|c|c|c|c|c|c|c|c|c|c|c|c|}
\hline \multicolumn{2}{|c|}{} & 1.0 & 0.8 & 0.63 & 0.5 & 0.4 & 0.32 & 0.25 & 0.2 & 0.16 & 0.1 & 0.08 & 0.05 & 0.02 & 0 \\
\hline & $5 / 5$ & $5 / 6$ & $5 / 8$ & $5 / 10$ & $5 / 12$ & $5 / 15$ & $5 / 20$ & $5 / 25$ & $5 / 30$ & $5 / 50$ & $1 / 12$ & $1 / 20$ & $1 / 50$ & 0 \\
\hline 1.0 & $5 / 5$ & 0 & 2 & 4 & 6 & 8 & 10 & 12 & 15 & 17 & 20 & 22 & 25 & 27 & 30 \\
\hline 0.8 & $5 / 6$ & 2 & 4 & 8 & 10 & 12 & 15 & 17 & 20 & 22 & 25 & 27 & 30 & 32 & 35 \\
\hline 0.63 & $5 / 8$ & 4 & 8 & 15 & 17 & 20 & 22 & 25 & 27 & 30 & 32 & 35 & 37 & 40 & 42 \\
\hline 0.5 & $5 / 10$ & 6 & 10 & 17 & 20 & 22 & 25 & 27 & 30 & 32 & 35 & 40 & 42 & 45 & 47 \\
\hline 0.4 & $5 / 12$ & 8 & 12 & 20 & 22 & 25 & 30 & 32 & 35 & 37 & 40 & 42 & 47 & 50 & 52 \\
\hline 0.32 & $5 / 15$ & 10 & 15 & 22 & 25 & 30 & 35 & 40 & 45 & 47 & 50 & 55 & 57 & 60 & 62 \\
\hline 0.25 & $5 / 20$ & 12 & 17 & 25 & 27 & 32 & 40 & 50 & 52 & 55 & 57 & 60 & 65 & 67 & 70 \\
\hline 0.2 & $5 / 25$ & 15 & 20 & 27 & 30 & 35 & 45 & 52 & 55 & 57 & 60 & 65 & 70 & 75 & 80 \\
\hline 0.16 & $5 / 30$ & 17 & 22 & 30 & 32 & 37 & 47 & 55 & 57 & 60 & 65 & 70 & 75 & 80 & 85 \\
\hline 0.1 & $5 / 50$ & 20 & 25 & 32 & 35 & 40 & 50 & 57 & 60 & 65 & 75 & 80 & 85 & 87 & 90 \\
\hline 0.08 & $1 / 12$ & 22 & 27 & 35 & 40 & 42 & 55 & 60 & 65 & 70 & 80 & 85 & 90 & 92 & 95 \\
\hline 0.05 & $1 / 20$ & 25 & 30 & 37 & 42 & 47 & 57 & 65 & 70 & 75 & 85 & 90 & 98 & 100 & 100 \\
\hline 0.02 & $1 / 50$ & 28 & 32 & 40 & 45 & 50 & 60 & 67 & 75 & 80 & 87 & 92 & 100 & 100 & 100 \\
\hline 0 & 0 & 30 & 35 & 42 & 47 & 52 & 62 & 70 & 80 & 85 & 90 & 95 & 100 & 100 & 100 \\
\hline
\end{tabular}

\section{Visual acuity}

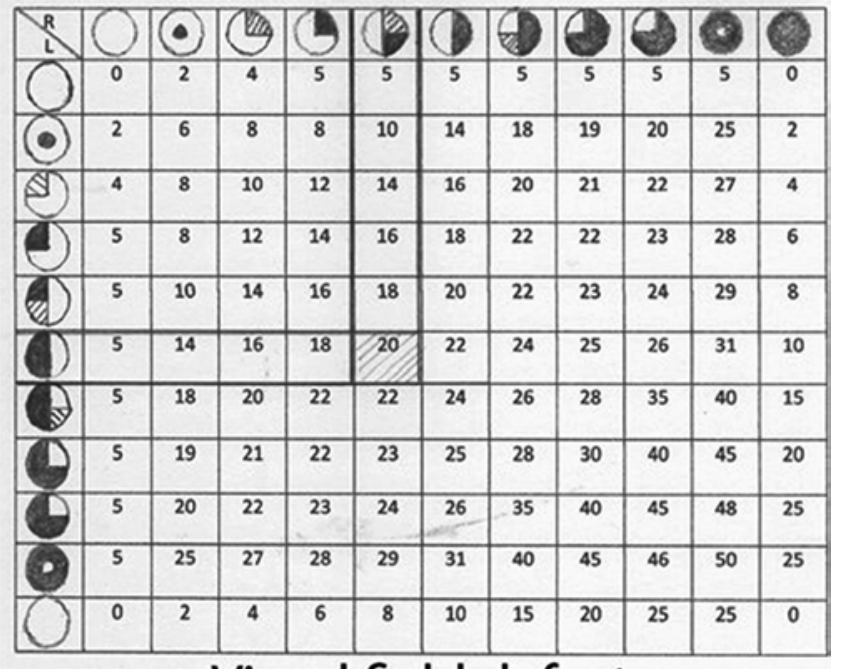

Visual field defect

FIG. 3. Illustrative case. Charts of visual acuity (left) and visual field defect (right) used for calculation of the VIS. The marked numbers provide an example of the calculation made in this case. The visual acuity was normal on both sides; the score of visual acuity of both eyes was 0 . The patient had complete temporal hemianopia in the left eye and partial temporal hemianopia in the right eye. The score of the field of vision was 20 . When the visual acuity score was added to the field defect score, the total was 20. This number represents the VIS.

\section{Volume of the Optic Chiasma}

The increase in the volume of the optic chiasma was also significantly correlated to the early postoperative improvement of the VIS and to the improvement of the VIS after 3 months (Spearman's rank correlation coefficient 0.63, significance level [2-tailed] 0.006).

\section{Duration of Symptoms}

The duration of the symptoms had a significant negative correlation to the decrease in the FA (Spearman's rank correlation coefficient -0.6 , significance level [2-tailed] 0.01 ) and a significant negative correlation to the improvement of the VIS (Spearman's rank correlation coefficient -0.49, significance level [2-tailed] 0.04).

\section{Age of the Patients}

The 2 patients who experienced visual deterioration were 65 and 72 years old. Nonetheless, there was no significant statistical correlation between the age of the patients and the visual outcome and FA changes found in this series.

\section{Illustrative Case}

A 56-year-old woman presented with progressive visual impairment that had started 4 weeks before admission. The ophthalmological evaluation revealed bitemporal hemianopia (complete on the left side and incomplete on the right side) without diminished visual acuity. To calculate the preoperative VIS we added the score of the visual defects of both eyes based on previously published special tables, ${ }^{12}$ which was 20 , to the score for visual acuity, which was 0 . The result of this summation was 20 (Fig. 3). The MRI revealed a pituitary adenoma with suprasellar extension and compression of the optic chiasma (Fig. 4A and
B). The preoperative hormonal profile showed secondary hypothyroidism and secondary hypogonadism.

The patient underwent tumor resection via the transsphenoidal approach under intraoperative MRI control. The volumetric T1-weighted images were fused with the DTI and the optic chiasma was segmented. The FA values and the volume of the optic chiasma were measured. The FA of the right cerebral peduncles was measured as a reference point. The surgery was performed and the tumor was resected (Fig. 4C and D). Immediately after tumor resection, the control MRI was performed while the patient's head was still fixed in the head fixator. The T1weighted postresection images were fused with the postresection DTI. The optic chiasma was segmented and the FA values and the volume were measured. The decrease of the average FA value of the optic chiasma was 0.16 . The volume of the optic chiasma increased as well. The postoperative ophthalmological evaluation revealed an incomplete left superior quadrantanopia with compete recovery of the rest of the visual field. There was no deterioration of the visual acuity. Accordingly, the postoperative VIS was 2 . After 3 months, the visual field defect recovered completely. The postoperative course was uneventful, without deterioration of the hormonal status. The histopathological examination of the tumor revealed a pituitary adenoma with weak immunoreactivity to luteinizing hormone (gonadotropinoma).

\section{Discussion}

Magnetic resonance imaging is the imaging modality of choice for preoperative evaluation of patients with suprasellar tumors. The MR images provide detailed structural information about the tumor, its extent, and its relation to the nearby structures. Diffusion tensor imaging is a de- 

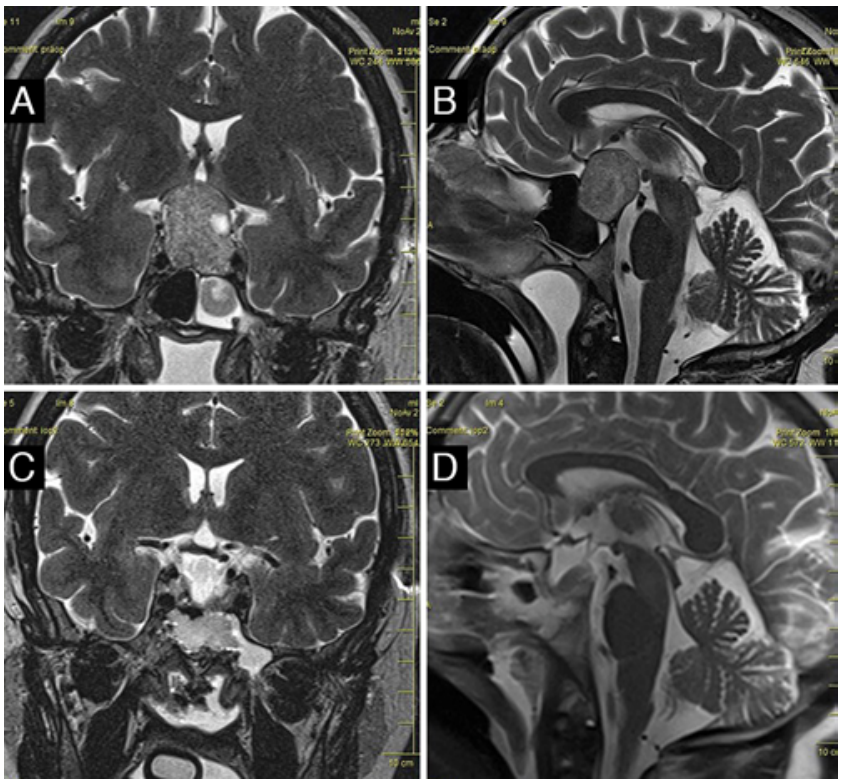

FIG. 4. A and B: Preoperative MR images of a pituitary adenoma that compressed the optic chiasma. C and D: Postoperative MR images showing the chiasma after tumor resection, which was performed via the transsphenoidal approach. Figure is available in color online only.

velopment of diffusion-weighted imaging that has longer acquisition times but provides more accurate quantitative information than diffusion-weighted imaging. DTI is a valuable tool in assessing the axonal architecture of white matter tracts by quantifying the amount of nonrandom water diffusion within nerve tissues in vivo, $7,8,10,21,22,28,30$, ${ }^{34,37}$ which is particularly important in the investigation of tumors in patients with subtle structural abnormalities. FA is one of the DTI indices that is usually used to investigate white matter changes. ${ }^{6,11,14,17}$ The degeneration of white matter tracts and/or the corresponding cortex results in a decrease of FA value due to the loss of the directionality of diffusion. ${ }^{3,9,11,14}$ DTI of the cranial nerves has also been evaluated in different pathological conditions. It has been applied, for example, in fiber tracking of the facial nerve in cases of vestibular schwannoma. ${ }^{13,36}$ The FA value has been also assessed in cases of trigeminal neuralgia caused by vascular compression, and can be correlated to the atrophic changes in the trigeminal nerve. ${ }^{24,25,27}$ DTI of the visual pathways has been used to evaluate the anatomy of these pathways in cases of different pathological conditions. ${ }^{16,23,31}$ The FA of the optic pathways has been evaluated in many pathological conditions. ${ }^{1,7,23}$ The advantage of the intraoperative MRI is to identify the residual tumor that necessitates further resection in some cases. 5,29,38 The structural changes in the visual pathways, including the change in the volume of the optic chiasma, can also be evaluated using intraoperative MRI.

In this study we measured the FA values of the optic chiasma obtained from the DTI sequences performed intraoperatively before and after decompression of the optic chiasma, and tested their correlation to the visual outcome. We also tested the correlation of the expansion of the volume of the optic chiasma to the visual outcome.
Furthermore, other possible predictors like the age of the patients and the duration of symptoms were tested.

Interestingly, the average FA values of the optic chiasma decreased significantly after decompression. This decrease in the average FA value was significantly correlated to the improvement of the visual status. The patients in whom the average FA values decreased more showed more improvement of the VIS after surgery. The decrease in the FA was significantly correlated to the VIS after 3 months. In the 2 patients who experienced deterioration of visual condition after surgery, the FA value was unchanged in one patient and decreased in the other patient. Interestingly, the patient who showed a decrease in the intraoperative FA value and expansion of the optic chiasma showed rapid improvement within 3 months. The VIS was unchanged in 2 patients 2 weeks after surgery, but the intraoperative average FA values decreased and the volume of the optic chiasma increased. These 2 patients showed improvement of the VIS after 3 months. Although the total number of patients with early visual deterioration or unchanged visual status was small, the available data showed that the decrease in the intraoperative FA could be associated with a delayed visual improvement after 3 months.

The preoperative average FA values did not significantly correlate to the preoperative VIS, nor did the postoperative FA values significantly correlate to the postoperative VIS. This is consistent with the previous observations that the FA values are variable among the population..$^{19,35,39}$ Thus, a single standalone FA value cannot predict the degree of visual impairment, but the change in the FA value can indicate an underlying pathological change.

We can explain this finding based on the fact that in a compressed optic chiasma the fibers are crowded and have high density. This can be associated with an increase in the anisotropy and thus an increase in the average FA value. When the optic chiasma is decompressed, the fiber overcrowding is reversed immediately and thus the anisotropy will reach a lower level and the FA as well. This was demonstrated in this series by the increase in the volume of the optic chiasma in 22 patients. This increase in the volume was significantly correlated to the decrease in the FA and to the improvement of the VIS. These changes reflected the direct early effect of decompression. On the other hand, in a patient with chronic compression the neural tissue shows permanent changes that cannot be completely reversed with decompression, and the FA value and the volume will either not change or will minimally change. The decrease in the FA is more sensitive and has a stronger correlation to the visual outcome because the FA can also detect changes in the microstructures of the optic chiasma.

Anik et al. reported a delayed increase in the FA of the affected bundles after decompression of the chiasma. This increase could be correlated to the visual outcome. ${ }^{1}$ Our study depicted the immediate change of the FA after decompression as it was measured based on intraoperative MRI; it reflected the early phase of recovery. In the study of Anik et al., the DTI was performed at a delayed point in time, which can reflect the delayed process of remyelination of the visual pathway and the delayed vi- 
TABLE 1. Correlation coefficient of the tested predictors of the visual outcome in 28 patients with suprasellar tumors

\begin{tabular}{lcc}
\hline \multicolumn{1}{c}{ Predictor } & $\begin{array}{c}\text { Correlation } \\
\text { Coefficient }\end{array}$ & $\begin{array}{c}\text { Significance } \\
\text { Level (2 tailed) }\end{array}$ \\
\hline Decrease in the FA & 0.76 & $<0.0001$ \\
\hline Expansion of optic chiasma & 0.63 & 0.006 \\
\hline Duration of symptoms & -0.6 & 0.01 \\
\hline Age & 0.16 & 0.47 \\
\hline
\end{tabular}

sual recovery. ${ }^{33}$ It has previously been described that the improvement happens in 2 phases with 2 different clinicopathological conditions. The first phase is the immediate improvement after surgery due to restoration of the conductivity after decompression. The second phase is a prolonged process of restoration of myelination and it may take years. ${ }^{15,33}$

The decrease in the intraoperative FA showed a more powerful correlation to the improvement of the VIS, followed by the expansion of the volume of the optic chiasma, and then by the duration of symptoms (Table 1). We could not find a correlation between the age of the patients and the visual outcome. Thus, the intraoperative decrease in FA value was also important as a predictor of delayed recovery in patients with a worsened or unchanged early VIS. A decrease in the intraoperative FA can also indicate a delayed improvement of the visual status, even in patients with unchanged or worsened postoperative visual status.

The value of the intraoperative application of the DTI in predicting the visual outcome has been previously discussed by Hajiabadi et al. ${ }^{16}$ The distance between the optic tracts based on fiber tracking seemed to be correlated to the visual outcome. There are other predictors of the visual outcome in patients with suprasellar tumors. The optical coherence tomography results were reported to be a strong predictor of the visual outcome. ${ }^{18}$ The use of optical coherence tomography scans was not assessed in our patients and we cannot provide a comparison with our predictors. However, this method could be an alternative when intraoperative MRI is not available. Other studies reported the preoperative visual status and the tumor size as predictors of the visual outcome. ${ }^{2,20,26}$ In this article we are providing 2 new predictors, the intraoperative decrease in the FA and the expansion of the volume of the optic chiasma.

Another potential application of this correlation is in the follow-up of patients with suprasellar tumors who are receiving conservative management, especially in uncooperative patients. An increase in the FA values in the followup images can indicate early or subtle clinical deterioration due to chiasma compression. It can give an impression about the degree of decompression and its efficiency, if partial tumor resection is planned. The decrease in the FA is associated with better long-term visual outcome, even in patients who show early postoperative visual deterioration.

\section{Conclusions}

Decrease in the FA and expansion of the optic chiasma after its decompression are early strong predictors of visual outcome. The correlation of the decrease in the FA and expansion of the optic chiasma to improvement of the VIS was stronger than the correlation with the duration of symptoms. The intraoperative decrease in the FA and expansion of the optic chiasma can also predict the delayed improvement of vision.

\section{Acknowledgments}

We thank Ms. U. Kabelitz, Mr. S. Wagner, and Mr. S. Mulamoottil from the intraoperative MRI team for their technical support. We thank Dr. R. Buslei (Department of Neuropathology, University of Erlangen-Nuremberg) for performing the histopathological examinations, and Drs. Moradi and Mohamadi for the ophthalmological workup of the patients.

\section{References}

1. Anik I, Anik Y, Koc K, Ceylan S, Genc H, Altintas O, et al: Evaluation of early visual recovery in pituitary macroadenomas after endoscopic endonasal transphenoidal surgery: Quantitative assessment with diffusion tensor imaging (DTI). Acta Neurochir (Wien) 153:831-842, 2011

2. Barzaghi LR, Medone M, Losa M, Bianchi S, Giovanelli M, Mortini P: Prognostic factors of visual field improvement after trans-sphenoidal approach for pituitary macroadenomas: review of the literature and analysis by quantitative method. Neurosurg Rev 35:369-379, 2012

3. Bauer CM, Heidary G, Koo BB, Killiany RJ, Bex P, Merabet LB: Abnormal white matter tractography of visual pathways detected by high-angular-resolution diffusion imaging (HARDI) corresponds to visual dysfunction in cortical/cerebral visual impairment. J AAPOS 18:398-401, 2014

4. Beppu T, Fujiwara S, Nishimoto H, Koeda A, Narumi S, Mori K, et al: Fractional anisotropy in the centrum semiovale as a quantitative indicator of cerebral white matter damage in the subacute phase in patients with carbon monoxide poisoning: correlation with the concentration of myelin basic protein in cerebrospinal fluid. J Neurol 259:1698-1705, 2012

5. Berkmann S, Schlaffer S, Nimsky C, Fahlbusch R, Buchfelder M: Intraoperative high-field MRI for transsphenoidal reoperations of nonfunctioning pituitary adenoma. J Neurosurg 121:1166-1175, 2014

6. Berman JI, Glass HC, Miller SP, Mukherjee P, Ferriero DM, Barkovich AJ, et al: Quantitative fiber tracking analysis of the optic radiation correlated with visual performance in premature newborns. AJNR Am J Neuroradiol 30:120-124, 2009

7. Cauley KA, Filippi CG: Diffusion-tensor imaging of small nerve bundles: cranial nerves, peripheral nerves, distal spinal cord, and lumbar nerve roots-clinical applications. AJR Am J Roentgenol 201:W326-W335, 2013

8. Clemm von Hohenberg C, Schocke MF, Wigand MC, Nachbauer W, Guttmann CR, Kubicki M, et al: Radial diffusivity in the cerebellar peduncles correlates with clinical severity in Friedreich ataxia. Neurol Sci 34:1459-1462, 2013

9. Dai H, Yin D, Hu C, Morelli JN, Hu S, Yan X, et al: Wholebrain voxel-based analysis of diffusion tensor MRI parameters in patients with primary open angle glaucoma and correlation with clinical glaucoma stage. Neuroradiology 55:233-243, 2013

10. Dong Q, Welsh RC, Chenevert TL, Carlos RC, Maly-Sundgren P, Gomez-Hassan DM, et al: Clinical applications of diffusion tensor imaging. J Magn Reson Imaging 19:6-18, 2004

11. Dudink J, Lequin M, van Pul C, Buijs J, Conneman N, van Goudoever J, et al: Fractional anisotropy in white matter tracts of very-low-birth-weight infants. Pediatr Radiol 37:1216-1223, 2007

12. Fahlbusch R, Schott W: Pterional surgery of meningiomas of the tuberculum sellae and planum sphenoidale: surgical 
results with special consideration of ophthalmological and endocrinological outcomes. J Neurosurg 96:235-243, 2002

13. Gerganov VM, Giordano M, Samii M, Samii A: Diffusion tensor imaging-based fiber tracking for prediction of the position of the facial nerve in relation to large vestibular schwannomas. J Neurosurg 115:1087-1093, 2011

14. Giordano M, Nabavi A, Gerganov VM, Javadi AS, Samii M, Fahlbusch R, et al: Assessment of quantitative corticospinal tract diffusion changes in patients affected by subcortical gliomas using common available navigation software. Clin Neurol Neurosurg 136:1-4, 2015

15. Gnanalingham KK, Bhattacharjee S, Pennington R, Ng J, Mendoza N: The time course of visual field recovery following transphenoidal surgery for pituitary adenomas: predictive factors for a good outcome. J Neurol Neurosurg Psychiatry 76:415-419, 2005

16. Hajiabadi M, Samii M, Fahlbusch R: A preliminary study of the clinical application of optic pathway diffusion tensor tractography in suprasellar tumor surgery: preoperative, intraoperative, and postoperative assessment. J Neurosurg 125:759-765, 2016

17. Ibrahim I, Tintera J, Skoch A, Jirů F, Hlustik P, Martinkova $\mathrm{P}$, et al: Fractional anisotropy and mean diffusivity in the corpus callosum of patients with multiple sclerosis: the effect of physiotherapy. Neuroradiology 53:917-926, 2011

18. Jacob M, Raverot G, Jouanneau E, Borson-Chazot F, Perrin G, Rabilloud M, et al: Predicting visual outcome after treatment of pituitary adenomas with optical coherence tomography. Am J Ophthalmol 147:64-70, 70.e1-70.e2, 2009

19. Kochunov P, Thompson PM, Lancaster JL, Bartzokis G, Smith S, Coyle T, et al: Relationship between white matter fractional anisotropy and other indices of cerebral health in normal aging: tract-based spatial statistics study of aging. Neuroimage 35:478-487, 2007

20. Lee S, Kim SJ, Yu YS, Kim YH, Paek SH, Kim DG, et al: Prognostic factors for visual recovery after transsphenoidal pituitary adenectomy. Br J Neurosurg 27:425-429, 2013

21. Lerner A, Mogensen MA, Kim PE, Shiroishi MS, Hwang DH, Law M: Clinical applications of diffusion tensor imaging. World Neurosurg 82:96-109, 2014

22. Likitjaroen Y, Meindl T, Friese U, Wagner M, Buerger K, Hampel H, et al: Longitudinal changes of fractional anisotropy in Alzheimer's disease patients treated with galantamine: a 12-month randomized, placebo-controlled, double-blinded study. Eur Arch Psychiatry Clin Neurosci 262:341-350, 2012

23. Lober RM, Guzman R, Cheshier SH, Fredrick DR, Edwards MS, Yeom KW: Application of diffusion tensor tractography in pediatric optic pathway glioma. J Neurosurg Pediatr 10:273-280, 2012

24. Lutz J, Thon N, Stahl R, Lummel N, Tonn JC, Linn J, et al: Microstructural alterations in trigeminal neuralgia determined by diffusion tensor imaging are independent of symptom duration, severity, and type of neurovascular conflict. J Neurosurg 124:823-830, 2016

25. Michelson G, Engelhorn T, Wärntges S, El Rafei A, Hornegger J, Doerfler A: DTI parameters of axonal integrity and demyelination of the optic radiation correlate with glaucoma indices. Graefes Arch Clin Exp Ophthalmol 251:243-253, 2013

26. Müslüman AM, Cansever T, Yılmaz A, Kanat A, Oba E, Çavuşoğlu H, et al: Surgical results of large and giant pituitary adenomas with special consideration of ophthalmologic outcomes. World Neurosurg 76:141-148, 63-66, 2011

27. Neetu S, Sunil K, Ashish A, Jayantee K, Usha Kant M: Microstructural abnormalities of the trigeminal nerve by diffusion-tensor imaging in trigeminal neuralgia without neurovascular compression. Neuroradiol J 29:13-18, 2016

28. Oppenheim C, Rodrigo S, Poupon C, Dumas de la Roque A,
Naggara O, Meder JF, et al: [Diffusion tensor MR imaging of the brain. Clinical applications.] J Radiol 85:287-296, 2004 (Fr)

29. Paterno' V, Fahlbusch R: High-field iMRI in transsphenoidal pituitary adenoma surgery with special respect to typical localization of residual tumor. Acta Neurochir (Wien) 156:463-474, 2014

30. Rollins NK: Clinical applications of diffusion tensor imaging and tractography in children. Pediatr Radiol 37:769-780, 2007

31. Salmela MB, Cauley KA, Andrews T, Gonyea JV, Tarasiewicz I, Filippi CG: Magnetic resonance diffusion tensor imaging of the optic nerves to guide treatment of pediatric suprasellar tumors. Pediatr Neurosurg 45:467-471, 2009

32. Sato Y, Ito K, Ogasawara K, Sasaki M, Kudo K, Murakami $\mathrm{T}$, et al: Postoperative increase in cerebral white matter fractional anisotropy on diffusion tensor magnetic resonance imaging is associated with cognitive improvement after uncomplicated carotid endarterectomy: tract-based spatial statistics analysis. Neurosurgery 73:592-599, 2013

33. Smith KJ, Blakemore WF, McDonald WI: The restoration of conduction by central remyelination. Brain 104:383-404, 1981

34. Sundgren PC, Dong Q, Gómez-Hassan D, Mukherji SK, Maly P, Welsh R: Diffusion tensor imaging of the brain: review of clinical applications. Neuroradiology 46:339-350, 2004

35. Turken A, Whitfield-Gabrieli S, Bammer R, Baldo JV, Dronkers NF, Gabrieli JD: Cognitive processing speed and the structure of white matter pathways: convergent evidence from normal variation and lesion studies. Neuroimage 42:1032-1044, 2008

36. Ung N, Mathur M, Chung LK, Cremer N, Pelargos P, Frew A, et al: A systematic analysis of the reliability of diffusion tensor imaging tractography for facial nerve imaging in patients with vestibular schwannoma. J Neurol Surg B Skull Base 77:314-318, 2016

37. Vedantam A, Eckardt G, Wang MC, Schmit BD, Kurpad SN: Clinical correlates of high cervical fractional anisotropy in acute cervical spinal cord injury. World Neurosurg 83:824828,2015

38. Vitaz TW, Inkabi KE, Carrubba CJ: Intraoperative MRI for transphenoidal procedures: short-term outcome for 100 consecutive cases. Clin Neurol Neurosurg 113:731-735, 2011

39. Wheeler-Kingshott CA, Trip SA, Symms MR, Parker GJ, Barker GJ, Miller DH: In vivo diffusion tensor imaging of the human optic nerve: pilot study in normal controls. Magn Reson Med 56:446-451, 2006

\section{Disclosures}

The authors report no conflict of interest concerning the materials or methods used in this study or the findings specified in this paper.

\section{Author Contributions}

Conception and design: Metwali, Fahlbusch. Acquisition of data: Metwali, Giordano, Kniese. Analysis and interpretation of data: Metwali. Drafting the article: Metwali, Giordano, Kniese. Critically revising the article: Metwali, Fahlbusch. Reviewed submitted version of manuscript: Metwali, Fahlbusch. Approved the final version of the manuscript on behalf of all authors: Metwali. Statistical analysis: Metwali. Study supervision: Fahlbusch.

\section{Correspondence}

Hussam Metwali, International Neuroscience Institute, RudolfPichlmayr-Straße 4, Hannover D-30625, Germany. email: drhussamm@yahoo.com. 